\title{
Stress et Spermatogénèse
}

\author{
Maurice Auroux
}

Biologie de la Reproduction et du Développement, CHU Bicêtre, 94270 Le Kremlin Bicêtre

\section{RESUME}

Le stress, dont le point de départ est cérébral, peut agir sur la spermatogénèse par les voies hormonale ou nerveuse. La voie hormonale aboutit à une baisse de production de LHRH, à une chute des récepteurs à $L H$ des cellules de Leydig et à une diminution de l'activité enzymatique $17 \alpha$ hydroxylase. Ainsi, en cas de stress important et prolongé, la sécrétion de testostérone diminue et la spermatogénèse peut en être affectée. La question de l'influence des stress de faible intensité reste posée.

La voie nerveuse comporte des fibres effectrices à catécholamines qui innervent les différentes parties du testicule. La destruction expérimentale de ces voies entraîne une régression de l'épithélium séminal. En outre, la destruction expérimentale du néocortex antérieur, chez le rat, provoque des altérations de la spermatogénèse. Les étages les plus élevés de la sphère nerveuse pourraient donc participer au contrôle de l'épithélium germinal.

On peut cependant faire l'hypothèse que c'est lorsque la production spermatique est basse qu'un stress momentané peut, en aggravant la situation, devenir nocif. Ainsi, l'impact du stress ou des facteurs psychiques sur la spermatogénèse pourrait n'être que relatif.
Mots clés : spermatogenèse, stress, testostérone, système nerveux.

La fatigue, les soucis, l'anxiété ou le stress sont souvent évoqués par les patients d'une consultation de stérilité masculine pour expliquer leur état. Leur répondre est difficile. Ce domaine est en effet assez flou alors que les perturbations du cycle féminin sont, pour les mêmes raisons, communément admises. Il est vrai que nous avons affaire à deux phénomènes différents. Le cycle menstruel se caractérise par la survenue d'événements discontinus et facilement repérables, tant dans leur expression normale que dans leur pathologie. Au contraire la spermatogénèse est un processus continu dont les variations quantitatives rendent difficile le repérage d'anomalies discrètes.

On soupçonne cependant depuis longtemps que l'organisme masculin n'échappe pas à ce qui touche si nettement le féminin. En effet, plusieurs articles ont déjà mentionné les liaisons négatives qui paraissent exister entre le stress et certains paramètres du spermogramme comme la numération, la mobilité et la morphologie des spermatozoïdes [3, 23, 24, 27]. A l'extrême, des situations très particulières comme l'attente d'une condamnation grave pourraient entraîner un arrêt de la spermatogénèse [34], celui-ci étant expérimentalement retrouvé chez le singe stressé par une immobilisation forcée [7]. Parallèlement, le stress du combat ou des affaires diminue- 
rait le niveau de testostérone circulante [23, 33]. Dans un domaine différent, les résultats de HARISSON et Coll. [17] suggèrent que le stress présenté par les hommes au moment d'une fécondation in vitro (FIV) entraîne une chute de certains des paramètres spermatiques, particulièrement nette en ce qui concerne le nombre total de spermatozoïdes par éjaculat, la mobilité et l'index de fertilité d'Eliasson. Dans la grande majorité des cas cela ne retentit pas sur le succès de la FIV mais, pour le petit nombre de spermes devenu très pathologiques, les auteurs notent une diminution des fécondations.

A côté de ces situations particulièrement aiguës existent les problèmes psychologiques ordinaires. S'il est classique de les assimiler à des stress d'intensité variable dont l'impact fluctue selon la nature des personnalités, leur étude est plus délicate [10]. C'est à ces événements de la vie courante que les patients font le plus souvent allusion. Dans cette perspective, on peut se demander si le rythme de plus en plus stressant de la vie moderne ne serait pas une des causes de la chute progressive de certains des paramètres spermatiques notée par CARLSEN et coll. depuis 50 ans en Europe [5] et par AugEr et coll. depuis 20 ans dans la région parisienne [2]. Bien entendu l'anxiété, la dépression peuvent diminuer l'appétit sexuel, mais cet aspect des choses est différent et nous ne l'envisagerons pas ici.

\section{LE STRESS}

S'il est aigu, le stress peut s'accompagner d'une diminution des réponses aux sollicitations de l'environnement, de culpabilité, de dépression, d'anxiété, de troubles du sommeil, de difficultés de mémorisation et de concentration. Toutes ces manifestations sont dues à des modifications métaboliques au centre desquelles se situent les catécholamines qui, selon l'expression de CANNON [4], sont "les hormones des situations critiques". Au cours des situations d'agression le système sympathique est donc activé, cela par la formation réticulée qui rassemble les stimulations venant de l'environnement via les organes des sens et le thalamus (sur l'animal décérébré, la stimulation de la substance réticulée entraîne la libération de catécholamines dans la circulation [20]).

Le point de départ du stress est cérébral. Ses effets secondaires empruntent des voies nerveuses et hormonales. Les voies nerveuses engendrent des effets immédiats, par exemple une accélération cardiaque ; dans cette perspective, mais sans qu'on puisse aujourd'hui préciser leur rôle au cours du stress, il est remarquable que des afférences végétatives innervent le testicule. Les voies hormonales sont commandées par l'axe hypothalamo-hypophysaire, interface entre le cerveau et le système hormonal. C'est par elles que nous commencerons cette étude car des travaux relativement récents ont montré que le stress perturbait les équilibres hormonaux impliqués dans la spermatogénèse.

\section{STRESS, HORMONES ET SPERMATOGENESE}

Le stress pourrait exercer un effet inhibiteur sur la spermatogénèse en diminuant le taux de la testostérone plasmatique. Cet effet a été vérifié avant les interventions chirurgicales [37], et il semble plus net chez les sujets jeunes que chez les sujets âgés [11]. Le stress de la vie courante aurait les mêmes conséquences [19]. Quels sont les mécanismes en cause?

Le stress entraîne une diminution du taux de la LH circulante. Cette diminution pourrait soit résulter d'une chute d'activité des neurones à LHRH [16] soit d'une altération de la réponse pituitaire [35]. De fait, le stress induit une sécrétion centrale de Corticotropin-Releasing Factor (CRF) [29], lequel augmente le taux des endorphines, qui inhibent la libération de dopamine [25], dont la chute entraîne à son tour une baisse 
de production du LHRH [14]. Récemment, d'ailleurs, on a envisagé que le CRF pourrait inhiber directement la sécrétion de $\mathrm{LH}$ [1, 12]. L'axe hypothalamo-hypophyso-surrénalien paraît donc impliqué dans ces phénomènes. Mais l'hypercortisolémie initiale liée au stress diminuerait aussi, directement, la sensibilité des cellules de Leydig à la $\mathrm{LH}$ en diminuant le nombre des récepteurs correspondants. Elle diminuerait en outre l'activité enzymatique $17 \alpha$ - hydroxylase $[13,38]$ c'est à dire l'un des maillons qui, de la progestérone ou de la prégnénolone, conduit à la testostérone.

Autrement dit le stress agirait aux niveaux central et périphérique.

Toutefois, les choses sont sans doute plus compliquées. En effet, les régulations locales de la stéroïdogénèse et de la spermatogénèse font intervenir de nombreux facteurs. Comme le montrent par exemple les travaux de MARSHALL [22], la production spermatique du singe semble directement proportionnelle au taux de testostérone intratesticulaire. Mais ce taux ne correspondant qu'à $82 \%$ du taux plasmatique normal, cela remet en question la notion classique qu'il faille de très fortes doses de testostérone pour initier ou maintenir la spermatogénèse. Chez le rat, d'ailleurs, $10 \%$ de la concentration intratesticulaire normale suffisent à la maintenir [9]. Autrement dit, la diminution de la testostérone au cours du stress ne suffit peut-être pas, chez l'homme, à perturber la spermatogénèse et cela d'autant moins que cette hormone semble peu intervenir dans la régulation des fonctions sertoliennes humaines [15] dont l'importance est considérable dans la spermatogénèse.

Néanmoins, l'absence totale de testostérone ne semble pas compatible avec une spermatogénèse normale $[6,21,22]$ et, dans cette mesure, on peut admettre qu'un stress, s'il déprime beaucoup la production de l'hormone, puisse être responsable d'une chute de la production.

\section{LES RELATIONS NEUROGONADIQUES DIRECTES}

A côté des facteurs hormonaux, les relations neurogonadiques directes pourraient, dans le cadre très général de la psychosomatique, jouer un rôle dans les modifications de la spermatogénèse. Une simple étude structurale [18] montre en effet que, grâce à une innervation locale qui intéresse les vaisseaux, les cellules glandulaires et les tubes séminifères, le SNC possède les moyens d'agir directement sur la gonade. L'innervation du testicule est ainsi assurée par les rameaux du plexus spermatique. On distingue :

- une innervation sensitive située dans la vaginale et l'albuginée avec des terminaisons libres et encapsulées,

- une innervation effectrice qui regroupe :

a) des filets nerveux au contact de complexes cellulaires sympathicotropes situés dans le corps d'Highmore,

b) des filets nerveux à catécholamines qui, chez l'homme, sont arrangés en plexus autour des ramifications vasculaires, à proximité de la membrane propre des tubes séminifères et au voisinage des cellules interstitielles,

c) des fibres amyéliniques de type végétatif qui se trouvent au contact de la membrane propre et même dans son épaisseur, entre les cellules contractiles. Une telle innervation devient particulièrement significative quand on sait le rôle de cette membrane dans les échanges métaboliques qui se produisent entre l'épithélium séminal et les capillaires péritubulaires.

Corrélativement, les travaux de CoUJARD [8] ont montré que, chez le cobaye, une destruction du ganglion sympathique prostato-vésiculo-déférentiel entraîne une régression de l'épithélium séminal. Les hormones gonadotropes, normalement produites, ne s'opposent pas à l'installation de ces lésions. 
La nature de l'action nerveuse directe.

Selon les auteurs qui l'admettent, l'influence directe du SNC sur la gonade serait de nature trophique et se manifesterait par le moyen de relais vasculaires. Un défaut de trophicité entraînerait un trouble de la réception gonadique aux stimulines. Cependant, compte tenu de ce qui vient d'être dit sur l'innervation du testicule, on peut aussi envisager que l'action du SNC intéresse directement les cellules de Leydig ou la membrane propre. Celle-ci contient de la mono-amino-oxydase, enzyme qui s'opposerait à la pénétration intratubulaire de la sérotonine [36], toxique pour la spermatogénèse [26]. On peut donc admettre qu'une afférence nerveuse directe puisse modifier ces équilibres locaux.

Les atrophies tubulaires des paraplégiques confirment l'influence du système nerveux sur la spermatogénèse, via les relais que l'on vient de décrire. On peut enfin se demander si la sphère cérébrale elle-même et, en particulier, ses niveaux les plus élevés, n'est pas capable d'influer sur la production spermatique. Des expériences anciennes semblent permettre de répondre affirmativement. SOULAIRAC et Coll [31, 32] ont en effet mis en évidence, chez le rat, que des lésions du néocortex antérieur provoquaient des altérations de la spermatogénèse. La structure de l'hypophyse glandulaire restant normale et l'administration de gonado-stimulines aux animaux expérimentés n'ayant pas d'effet réparateur, les auteurs concluaient à une participation nerveuse au contrôle de la différenciation de l'épithélium germinal. Ces résultats expérimentaux correspondent bien entendu à des situations très aiguës qui n'ont rien de commun avec l'anxiété ou les soucis quotidiens. Ils montrent cependant que les étages les plus élevées de la sphère nerveuse peuvent, exercer une influence sur la spermatogénèse, que les relais soient nerveux ou hormonaux.

\section{LES FLUCTUATIONS DE LA PRODUCTION SPERMATIQUE}

Il convient maintenant de situer les effets du stress relativement aux fluctuations intra et inter-individuelles de la production spermatique. On sait que ces fluctuations sont considérables. La numération des spermatozoïdes, par exemple, est le paramètre le plus variable de l'organisme : son coefficient de variation chez un même individu est de 0,40 et, entre les individus, il est de 0,69 . A titre de comparaison, les coefficients de variations inter-individuels des autres paramètres organiques varient de 0,05 à 0,20 (terision artérielle, cholestérolémie, durée de vie etc.) [30]. La numération individuelle peut ainsi passer de 100 millions $/ \mathrm{ml}$ à 2 millions, remonter à 50 , redescendre à 20 etc [28], ces fluctuations étant semble-t-il indépendantes du taux de testostérone assez constant nécessaire à une spermatogénèse normale, et peut-être en rapport avec les régulations locales. On peut donc supposer que l'effet d'une chute brève du taux hormonal au dessous de ce seuil ne sera préjudiciable, en aggravant la situation, que s'il concerne les périodes où la production spermatique est basse. On peut tenir un raisonnement analogue pour les facteurs nerveux. Comme, chez un homme normal, ces périodes sont courtes, la probabilité d'une répercussion sur la fertilité est faible. En revanche, si l'homme considéré fluctue dans des zones basses, de telles périodes sont plus fréquentes et la probabilité de cette répercussion augmente. Ainsi, l'impact du stress ou des facteurs psychiques sur la spermatogénèse ne serait-il que relatif.

\section{CONCLUSION}

Un stress de grande intensité peut déprimer la spermatogénèse et, si l'influence de facteurs psychiques d'intensité moindre, comme l'anxiété, n'est pas encore prouvée, elle est vraisemblable. Dans cette perspective, et indépendamment des facteurs hormo- 
naux, il faut insister sur l'existence des relations neuro-gonadiques directes, qui pourraient expliquer l'influence sur la spermatogénèse des étages cérébraux les plus élevés. Il faut cependant relativiser l'importance de ces facteurs, la production des gamètes y étant peut-être d'autant moins sensible qu'elle est importante. La marge de sécurité serait donc assez grande, correspondant sans doute, au delà de la fragilité psychologique de l'individu, à la solidité des mécanismes de conservation de l'espèce.

\section{REFERENCES}

1. Almeida OFX., Nikolorakis KE., Herz A. : Evidence for the involvement of endogenous opioids in the inhibition of luteinizing hormone by corticotropin-releasing factor. Endocrinology, 1988, 122, 1034-1041.

2. Auger J., Czyglik F., Kunstmann JM., Jounnnet P. : Significant decrease of semen characteristics of fertile men from the Paris area during last 20 years. Hum. Reprod., 1994, 9, Suppl. 4, 175.

3. Bentz H. : Psychology of male infertility : a literature survey. Int. J. Androl. 1985, 8, 325-336.

4. CANNoN WB. : Traumatic shock, 1 vol, Appleton, New York, 1923.

5. Carlsen E., Giwercman A., Keiding N., SkakkeBAEK NE. : Evidence for decreasing quality of semen during past 50 years. Brit. Med. J., 1992 , 305, 609-613.

6. Chowdhury AK., Tcholakian RK. : Effects of various doses of testosterone propionate on intratesticular and plasma testosterone levels and maintenance of spermatogenesis in adult hypophysectomised rats. Steroids, 1979, 34, 151-162.

7. Cockett ATK., Elbadawi A., Zemuanis R., Adey WR. The effects of immobilization on spermatogenesis in subhuman primates. Fertil. Steril. 1970, 21, 610-614.

8. COUJARD : Le facteur nerveux périphérique. Les incidences sur la spermatogénèse, in "La fonction spermatogénétique du testicule humain". Masson ed., Paris, 1958, 91-105.

9. Cunningham GR., Huckins C. : Persistence of complete spermatogenesis in the presence of low intratesticular concentrations of testosterone. Endocrinology, 1979, 105, 177-186.

10. CZYBA JC., CLEMENT JL. : Evaluation des facteurs psychogènes dans l'infertilité masculine. Les colloques de l'Inserm : Facteurs de la fertilité humaine. A. SPIRA, P. JOUANNET Eds. Inserm, 1981, 103, pp 453-460.
11. Deslypere JP., Vermeulen A. : Leydig cell function in normal men : effect of age, life style, residence, diet and activity. J. Clin. Endocrinol. Metab. 1984, 59, 955-962.

12. Dufau ML., Ulisse S., Khanum A., Buckzo E., Kitamura M., Fabbri A., Namiki M. : LH action in the Leydig cell : modulation by angiotensin II and corticotropin releasing hormone and regulation of $p$ 45017 a m RNA. J. Steroid Biochem. 1989, 34, 205-217.

13. Doerr P., Pirke KM. : Cortisol-induced suppression of plasma testosterone in normal adult males. J. Clin. Endocrinol. Metab. 1976, 43, 622-629.

14. DunBar R. : Stress as a good contraceptive. New Scientist, 1985, 17, 16-18.

15. Foucault P., Carreau S., Kuczinsky W., GulllauMin JM., BARdos P., Drosdowski MA. : Human Sertoli cells in vitro. Lactate, estradiol $-17 \mathrm{~B}$ and transferin production. J. Androl., 1992, 13, 361367.

16. Gray GD., Smith ER., Damassa DA., Ehrenkranz JR., DAVIDSON JM. : Neuroendocrine mechanisms mediating the suppression of circulating testosterone levels associated with chronic stress in male rats. Neuroendocrinology, 1978, 25, 247-256.

17. Harrisson KL., Callan VJ., Hennessey JF. : Stress and semen quality in an in vitro fertilization program. Fertil. Steril., 1987, 48, 633-636.

18. Hodson M. : The nerves of the testis, epididymis ans scrotum in testis, development, anatomy and physiology, Johnson and Vandemarked eds. Acad. Press., 1970, London, 47-99.

19. Lopez-Calderon A., Ariznavaretta C., GonzalesQuijano MI., Tresguerres JA., Calderon MD. : Stress induced changes in testis function. J. Steroid. Biochem. Molec. Biol. 1991, 40, 473-479.

20. MARLEY E. : Release of an adrenalin-like substance by electrical stimulation of the brain stem. In Ciba symposium on adrenergic mechanism JR. VANE Ed. Churchill, Londres 1960, 424-431.

21. Marshall GR., Wickings EJ., Luedecke DK., NiesCHLAG E. : Stimulation of spermatogenesis in stalksectioned rhesus monkeys by testosterone alone. J. Clin. Endocrinol. Metab., 1983, 57, 152-159.

22. Marshall GR., Wickings EJ., Nieschlag E. : Testosterone can initiate spermatogenesis in an immature non human primate, Macaca fascicularis. Endocrinology 1984, 114, 2228-2233.

23. MC GRADY AV : Effects of psychological stress on male reproduction : a review. Arch. Androl., 1984, $13,1-7$.

24. Moghissi KS., Wallach EE. : Unexplained infertility. Fertil. Steril. 1983, 39, 5-21. 
25. Nikolorakis KE., Almeida OF., Hertz A. : Stimulation of hypothalamic $B$ endorphin and dynorphin release by corticotropin-releasing factor (in vitro). Brain Res., 1986, 399, 152-155.

26. O'STEEN WK. : Sertonin and Histamine : Effect of a single injection on the mouse testis and prostate Gland. Proc. Soc. Exp. Biol. Med., 1963, 113, 161163.

27. Palti Z. : Psychogenic male infertility. Psychosom Med. 1969, 31, 326-330.

28. PAULSEN CA. : In : Who laboratory manual for the examination of human semen and semen cervical mucus interaction. World Health Organisation, Cambridge University Press, Cambridge, 1987, p 3.

29. Plotsky P., Vale W. : Hemmorrhage-induced secretion of corticotropin releasing factor like immunoreactivity into the rat hypophysial portal circulation and its inhibition by glucocorticoids. Endocrinology, 1984, 114, 164-169.

30. SchWARTz D. : Variations physiologiques du sperme. Rev. Prat., Paris, 1983, 33, 3109-3114.

31. Soulairac A., Soulairac ML. : Le contrôle nerveux de la spermatogénèse. Path. Biol., 1963, 11, 12101213.

32. Soulairac A, Soulairac ML. : Contrôle nerveux central extra hypophysaire de la fonction testiculaire. Acta Endoc, 1967, 8, 37-47.

33. Steeno OP., Pangkahila A. : Occupational influences on male fertility and sexuality. Andrologia $1984,16,5-22$.

34. STiEve H. : Der Einfluss des nevensystems ouf bau und tatigkect der geschlechtsorgane des menschen. Stuttgart, Thieme, 1952. Cité in HARRISSON and al. 1987.

35. Suter DE., Schwartz NB. : Effects of glucocorticoilds on secretion of luteinizing hormone and follicle stimulating hormone by female rat pituitary cells in vitro. Endocrinology, 1985, 117, 849-854.

36. URry RL., Dougherty KA., Ellis LC. : Alterations with age in rat seminiferous tubule monoamine oxidase activity when compared with whole testicular tissue (38636). Proc. Soc. Exp. Biol. Med., $1975,148,805-807$.

37. WANG C, Chan Y., YEUNG RT. : Effect of surgical stress on pituitary testicular function. Clin. Endocrinol (Oxf.) 1978, 9, 255-266.

38. Welsh TH., Bambino TH., Hsueh AJW : Mechanism of glucocorticoild induced suppression of testicular androgen biosynthesis in vitro. Biol. Reprod., 1982, 27, 1138-1146.

\section{ABSTRACT \\ Stress and spermatogenesis}

\section{Auroux}

Stress, which originates in the brain, can influence spermatogenesis hormonally or via the nervous system. The hormonal route commences with the central secretion of CorticotrophinReleasing Factor, leading to a fall in LHRH production, a decrease in Leydig cell $\mathrm{LH}$ receptors and a decrease in 17 a hydroxylase activity. Thus, in the case of major, prolonged stress, testosterone secretion falls, which in turn affects spermatogenesis. However, given that the testosterone threshold required for normal seminiferous epithelium function is significantly less than the mean circulating level of this hormone, the importance of low intensity stress remains unknown.

The nervous route involves catecholaminergic fibres which, in the testis, innervate the Highmore corpus, the vessels, the area adjacent to the Leydig cells, and the basement membrane of seminiferous tubules. The experimental destruction of these fibres leads a regression of the seminiferous epithelium. Moreover, the experimental ablation of the rat anterior neocortex leads to changes in spermatogenesis. Therefore, given that the endocrine system does not seem to be involved in these changes, these results indicate that the highest level of the nervous system may participate in the controlling the germinal epithelium which, all things considered, would tend to support psychosomatic influences.

However, given that the number of spermatozoa varies significantly between ejaculate and independantly of the level of testosterone secretion necessary for normal spermatogenesis, it may be hypothesized that it is only when sperm production is low that 
temporary stress, in aggravating the situation, becomes deleterious to spermatogenesis. Since, under normal conditions, such periods are short, the role of the influence of stress on spermatogenesis can only be relative. Nevertheless, if variations occur during permanently low sperm production, the likelihood of negative effects is increased. Consequently, the impact of stress or psychological factors on spermatogenesis might well depend upon particular circumstances.

Key-Words : spermatogenesis, stress, testosterone, nervous system. 